\title{
POPULATION STUDY ARTICLE Body fat indicators perform better than body mass index in identifying abnormal lipid profiles in boys but not in girls
}

Haibo $\mathrm{Li}^{1}$, Tao Huang ${ }^{2}$, Junting $\mathrm{Liu}^{3}$, Yinkun $\mathrm{Yan}^{3}$, Xiaoyuan $\mathrm{Zhao}^{3}$, Pei Xiao ${ }^{1}$ and Jie $\mathrm{Mi}^{1,4}$, on behalf of the China Child and Adolescent Cardiovascular Health $(\mathrm{CCACH})$ Collaboration Group

BACKGROUND: BMI as a body weight indicator, may inadequately represent the biological effect of body fat on lipid profiles. This study aims to assess whether body fat indicators were superior to BMI for recognizing children with dyslipidemia.

METHODS: A nationwide cross-sectional study involving 8944 pediatric participants aged 6-18 years. Measures of fat mass index (FMI), fat mass percentage (FMP), BMI, and four lipid profiles were obtained.

RESULTS: Among boys, the standard multi-linear regression coefficients of FMI for TC, LDL-C, and TG were higher than those of BMI $(P<0.01)$, but not for HDL-C. Also, the prevalence ratios and area under curves (AUCs) of excess fat classified by FMI for specific abnormal lipid profiles (except for HDL-C) were greater than overweight classified by BMI. The AUCs for detecting children with abnormal TC, LDL-C, and TG of FMI-based excess fat were 3.9\%, 5.6\%, and 2.8\% higher than those of BMI-based overweight, respectively, all $P<0.01$. Among girls, the associations of BMI with lipid profiles were substantially similar to FMI. All these results were almost identical when FMP was used instead of FMI.

CONCLUSIONS: DXA measured body fat performs better than BMI in identifying abnormal lipid profiles in boys but not in girls.

Pediatric Research (2019) 85:617-624; https://doi.org/10.1038/s41390-019-0287-x

\section{INTRODUCTION}

Childhood lipid disorders track into adulthood and predict adult dyslipidemia, ${ }^{1-3}$ which has been associated with an increased burden of atherosclerosis and risk of cardiovascular diseases (CVDs). ${ }^{4,5}$ Therefore, it is essential to identify children at high risk for abnormal lipids.

The association between high childhood adiposity and abnormal lipid concentrations are well documented. ${ }^{6-9}$ The American Academy of Pediatrics (AAP) and the National Heart, Lung, and Blood Institute (NHLBI) has recommended fasting lipid profile screening for children with overweight (estimated by body mass index $(\mathrm{BMI}) \geq 85$ th), and either dietary intervention or pharmacologic management may be warranted. ${ }^{10,11}$ Thus, a child's BMI may influence clinical decisions, BMI measures that exceed the normal range may trigger clinical intervention strategies.

Nevertheless, the American Association of Clinical Endocrinologists and American College of Endocrinology (AACE/ACE) had proposed in 2014: "The thrust of the final recommendations is to recognize that obesity is a complex, adiposity-based chronic disease". ${ }^{12}$ BMI as a body weight indicator does not distinguish between fat and lean mass and has limitations in estimating adiposity. ${ }^{13}$ Importantly, with increasing BMl girls develop more total fat and fat deposits in the arms and legs, whereas boys develop more total lean and muscle mass during the pubertal period. $^{14,15}$. Thus, BMI may inadequately represent the biological effect of body fat on lipid profiles. ${ }^{16,17}$ Also, it is not uncommon for a child with a BMI within the normal range to have dyslipidemia. ${ }^{18}$ The above studies call for a more precise approach to assess children's adiposity and its relation with lipid profiles in clinical practice. Currently, little is known on whether directly measured adiposity indexes are better than BMI to discriminate children with abnormal lipid profiles.

In the current study, data from a national wide sample of China youths was used, to estimate the relation between adiposity and lipid concentrations more precisely, and to assess whether fat mass index (FMI) and fat mass percentage (FMP) are superior to $\mathrm{BMI}$ regarding the ability to identify children with abnormal lipid profiles.

\section{METHODS}

Participants

The China Child and Adolescent Cardiovascular Health (CCACH) study was a large, nationwide and multicenter observational study conducted from 2013 to 2015, designed to examine cardiovascular health, body composition and bone mineral density among Chinese children and adolescents. Details of the design and methods of the CCACH study have been described previously. ${ }^{19}$ In the present study, we excluded participants who had missing data on BMI $(n=75)$ and serum lipid $(n=698)$ measures and those with diabetes or kidney or thyroid disease $(n=37)$. A total of 8944 $(91.7 \%)$ children ( 4486 boys and 4458 girls) aged 6-18 years with body fat data were included in the present study. The study was approved by the Research Ethics Committee of the Capital Institute of Pediatrics of China. The written informed consent

\footnotetext{
${ }^{1}$ Department of Epidemiology, Capital Institute of Pediatrics; Graduate School of Peking Union Medical College, Beijing, China; ${ }^{2}$ Department of Epidemiology and Biostatistics, School of Public Health, Peking University, Beijing, China and ${ }^{3}$ Department of Epidemiology, Capital Institute of Pediatrics, Beijing, China

Correspondence: Jie Mi (jiemi@vip.163.com)

${ }^{4}$ Present address: Beijing Children's Hospital, Capital Medical University, National Center for Children's Health, 56 Nalishi Road, Xicheng District, Beijing 100045, China
}

Received: 4 September 2018 Revised: 11 November 2018 Accepted: 1 January 2019

Published online: 17 January 2019 
documents for children were obtained from their parents or guardians.

\section{Questionnaire}

Dietary behaviors for the past 12 months were assessed by using a food frequency questionnaire. A healthy diet was defined to include the following 5 components: (1) bean-curd or soybean products ( $\geq 1$ time/day); ( 2 ) fruits and vegetables ( $\geq 1$ time/day); ( 3 ) fish or fish products ( $\geq 1$ time/week); (4) sugar-sweetened beverage ( $<1$ time/week); and (5) salty snacks ( $<1$ time/day), participants were classified as poor or intermediate diet (0-3 components), and ideal diet (4-5 components) based on the number of components they achieved. Ideal physical activity (PA) was defined by daily frequencies of 30-min moderate or vigorous PA beyond school physical education. ${ }^{20}$ Smoking and drinking status was categorized as never, attempt, or current.

Anthropometric measurement

Height was measured using wall-mounted stadiometers without shoes, degree of accuracy was $0.1 \mathrm{~cm}$. Weight was measured using beam scales with light indoor clothing and without shoes, degree of accuracy was $0.1 \mathrm{~kg}$. BMI was calculated as weight $(\mathrm{kg})$ divided by the square of height $\left(\mathrm{m}^{2}\right)$. BMI sex- and age-specific $z$-scores were calculated, and the World Health Organization (WHO) child growth standard ${ }^{21}$ was also used to classify BMI as nonoverweight and overweight (including obesity).

\section{Body fat measurement}

The whole-body dual-energy X-ray absorptiometry (DXA) was performed using Hologic Discovery (A,W and Wi) fan-beam densitometers (Hologic, Bedford, Massachusetts). Hologic Apex software Version 4.0 was used to analyze DXA scans. The coefficient of variation (CV) was used as the quality control procedure. CVs of A, W and Wi were $0.471 \%, 0.302 \%$, and $0.358 \%$, respectively. Quality control was maintained throughout the DXA data collection and scan analysis according to International Society for Clinical Densitometry (ISCD) recommendations. ${ }^{22}$ FMI was calculated as total fat mass $(\mathrm{kg})$ divided by the square of height $\left(\mathrm{m}^{2}\right)$, and FMP was calculated as total fat mass $(\mathrm{kg})$ divided by the weight $(\mathrm{kg})$. Excess fat was defined as FMI or FMP greater than or equal to the sex- and age-specific internal smoothed 75th percentile, ${ }^{19}$ respectively.

\section{Lipid measurement}

After an overnight fast of at least $12 \mathrm{~h}$, blood samples were collected from participant's antecubital vein in the morning and then were transfused into vacuum tubes containing ethylenediamine tetraacetic acid. Blood specimens were centrifuged at $2000 \times g$ for $10 \mathrm{~min}$ within $1 \mathrm{~h}$ of the collection at room temperature, and then aliquoted and frozen at $-80^{\circ} \mathrm{C}$. Plasma specimens collected in each center were shipped by air in dry ice to the central clinical laboratory of Capital Institute of Pediatrics in Beijing, where the specimens were stored at $-80^{\circ} \mathrm{C}$ until laboratory assays were performed. Total cholesterol (TC), triglycerides (TG), low-density lipoprotein cholesterol (LDL-C) and highdensity lipoprotein cholesterol (HDL-C) were analyzed by the direct method (Sekisui Medical Inc., Tokyo, Japan). The intra-assay CVs for TC, LDL-C, TG, and HDL-C were 1.59\%, 1.63\%, 1.92\%, and $1.18 \%$ respectively, while the inter-assay CVs were $1.87 \%$, $2.79 \%$, $1.95 \%$, and $2.38 \%$.

Abnormal lipid concentrations were defined as follows: $\mathrm{TC} \geq$ $200 \mathrm{mg} / \mathrm{dL}, \mathrm{LDL}-\mathrm{C} \geq 130 \mathrm{mg} / \mathrm{dL}$, and TG $\geq 110 \mathrm{mg} / \mathrm{dL}$ (if $<10$ years) or $\geq 130 \mathrm{mg} / \mathrm{dL}$ (if $\geq 10$ years), were considered above normal levels. $\mathrm{HDL}-\mathrm{C}<40 \mathrm{mg} / \mathrm{dL}$ was considered below normal levels. ${ }^{10,11}$

Statistical analysis

Measurement data were expressed as Mean (SD) or medians (interquartile range), differences among two groups were
Table 1. Characteristics of the study pediatric population aged 6-18 years by sex, 2013-2015

\begin{tabular}{llllr}
\hline Variables & Overall & Boys & Girls & $P$-value \\
\hline Sample size, $n$ & 8944 & 4486 & 4458 & \\
Age, y & $12.8 \pm 3.7$ & $12.7 \pm 3.7$ & $13.0 \pm 3.8$ & $<0.001$ \\
BMI, kg/m ${ }^{2}$ & $19.7 \pm 4.1$ & $20.1 \pm 4.4$ & $19.4 \pm 3.7$ & $<0.001$ \\
FMI, kg/m ${ }^{2}$ & $6.0 \pm 2.5$ & $5.6 \pm 2.6$ & $6.4 \pm 2.2$ & $<0.001$ \\
FMP, \% & $29.5 \pm 7.3$ & $26.8 \pm 7.7$ & $32.3 \pm 5.7$ & $<0.001$ \\
TC, mg/dL & $148.2 \pm 29.0$ & $146.5 \pm 28.7$ & $149.9 \pm 29.1$ & $<0.001$ \\
LDL-C, mg/dL & $83.3 \pm 24.1$ & $82.3 \pm 23.6$ & $84.3 \pm 24.4$ & $<0.001$ \\
TG, mg/dL & $57.6(40.7$, & $54.9(38.9$, & $59.3(42.5$, & $<0.001$ \\
& $80.6)$ & $80.4)$ & $81.5)$ & \\
HDL-C, mg/dL & $54.3 \pm 11.1$ & $53.3 \pm 11.0$ & $55.4 \pm 11.1$ & $<0.001$ \\
Overweight classified & $2478(27.7)$ & $1581(35.2)$ & $897(20.1)$ & $<0.001$ \\
by BMI, $n$ (\%) & & & & \\
FMI $\geq 75$ th, $n$ (\%) & $2697(30.2)$ & $1406(31.3)$ & $1291(29.0)$ & 0.015 \\
FMP $\geq 75$ th, $n$ (\%) & $2566(28.7)$ & $1311(29.2)$ & $1255(28.2)$ & 0.272 \\
High TC, $n$ (\%) & $420(4.7)$ & $190(4.2)$ & $230(5.2)$ & 0.044 \\
High LDL-C, $n$ (\%) & $337(3.8)$ & $146(3.3)$ & $191(4.3)$ & 0.012 \\
High TG, $n$ (\%) & $721(8.1)$ & $384(8.6)$ & $337(7.6)$ & 0.089 \\
Low HDL-C, $n$ (\%) & $728(8.1)$ & $438(9.8)$ & $290(6.5)$ & $<0.001$ \\
Never smoked, $n$ (\%) & $8204(91.7)$ & $3949(88.0)$ & $4255(95.4)$ & $<0.001$ \\
Never drank, $n$ (\%) & $6990(78.2)$ & $3362(74.9)$ & $3628(81.4)$ & $<0.001$ \\
Ideal diet, $n(\%)$ & $2740(30.6)$ & $1422(31.7)$ & $1318(29.6)$ & 0.030 \\
Ideal physical activity, & $2620(29.3)$ & $1525(34.0)$ & $1095(24.6)$ & $<0.001$ \\
$n$ (\%) & & & & \\
\hline BM body mass index, & & & \\
\hline
\end{tabular}

$B M I$ body mass index, FMI fat mass index, FMP fat mass percentage, $H D L-C$ high-density lipoprotein cholesterol, IQR interquartile range, LDL-C lowdensity lipoprotein cholesterol, TC total cholesterol, TG triglycerides

Measurement data were expressed as mean $\pm \mathrm{SD}$ or medians (IQR), differences among genders were analyzed by Student's $t$-test or Mann-Whitney $U$ test. Qualitative data were described as number of subjects (\%) and analyzed using Chi-square $\left(x^{2}\right)$ test as indicated

analyzed by Student's $t$-test or Mann-Whitney $U$ test. The TG levels were positively skewed and thus natural log-transformed to normalize their distribution. Qualitative data were described as number of subjects (\%) and analyzed using Chi-square $\left(x^{2}\right)$ test. Due to the characteristics are different between boys and girls, we performed the analysis by sex.

The pubertal stage was not measured in the $\mathrm{CCACH}$ study. According to previously Chinese children's studies reported the median ages at attainment of testicular volume of $4 \mathrm{~mL}$ or greater in boys ${ }^{23}$ and Tanner stage 2 of breast development in girls, ${ }^{24}$ age in here was divided into two groups: (1) boys $<11$ years, girls $<10$ years (representing pre-puberty); (2) boys $\geq 11$ years, girls $\geq 10$ years (representing puberty and post-puberty), in an effort to reflect the pubertal period.

To assess the relation of BMI, FMI, and FMP with lipid concentrations, sex (and age)-specific correlation analyses and multivariable linear regression models were used adjusted for possible confounding effects of age, study center, smoking, alcohol drinking, diet, and physical activity. We further conducted analyses with mutual adjustment for the BMI and FMI/FMP to evaluate their independent associations with lipid profiles (simultaneous models). To examine sex and age differences in the relationships, multivariable models that included a variable to represent the interaction between adiposity indicators and sex or age groups were used. In all linear models, BMI, FMI, FMP, and lipid concentrations were used on their SD scale (since there is a wide age range, sex- and age-specific $z$-scores were calculated, for TG $z$-score of log-transformed values), so that the magnitude of 

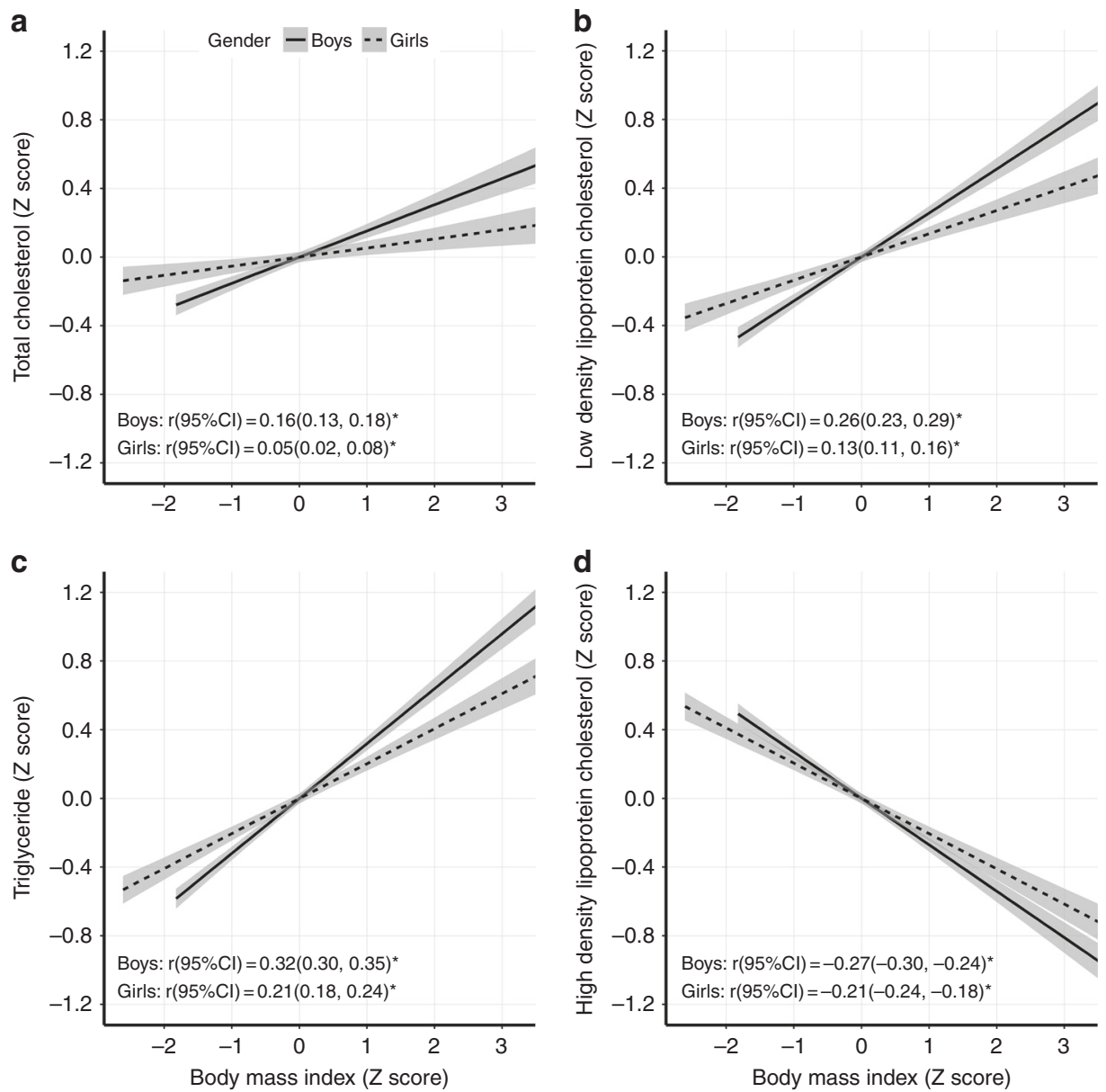

Fig. 1 Plot of relationships among body mass index and lipid profiles by sex in Chinese children and adolescents aged 6-18 years. Body mass index shown in relationship to total cholesterol (a), low-density lipoprotein cholesterol (b), triglyceride (c), and high-density lipoprotein cholesterol (d). Solid lines denote boys, dotted lines denote girls, shading indicate $95 \% \mathrm{Cl} . r(95 \% \mathrm{Cl})$, correlation coefficient and $95 \% \mathrm{Cl}^{*}{ }^{*}$ all $P<0.05$. For triglyceride, $z$-score of log-transformed values

regression coefficients between models was standardized and directly comparable. The bootstrap method also was used to test the equivalence of coefficients between models. ${ }^{25}$ Furthermore, log-binomial regression models and receiver operating characteristic curves (ROCs) were conducted to assess the capacity of BMI and body fat status for recognizing specific abnormal lipid profiles, and COPY method that was primarily developed to overcome non-convergence of log-binomial regression models. ${ }^{26}$

Statistical significance was defined as a two-tailed $P$ value $\leq$ 0.05 . Data management and all analyses were performed using $R$ software, version 3.4 .3 (http://www.R-project.org).

\section{RESULTS}

Subject characteristics

In 8944 children aged 6-18 years, when BMI was used to define weight status, $27.3 \%$ children ( $33.4 \%$ boys, $21.1 \%$ girls) were overweight (including obesity). There were $28.7 \%$ children $(29.2 \%$ boys, $28.2 \%$ girls) who had excess fat defined by FMI, and the percentages were similar for FMP. Approximately $18.8 \%$ of the total sample had dyslipidemia (4.7\% high TC, 3.8\% high LDL, $8.1 \%$ high TG, and $8.1 \%$ low HDL). The prevalence of adverse TC and LDL was greater in girls than boys, whereas boys had higher adverse $\mathrm{HDL}$ rate $(P<0.05)$. No statistically significant sex difference in high TG was found $(P=0.089$, Table 1$)$. BMI increased with age for both genders. FMl constantly increased during 6-18 years for girls, while increased until about 11 years and decreased after that for boys. FMP did not change essentially before 10-11 years for both genders, after that, FMP increased for girls but decreased for boys (Supplementary Figure S1 (online)).

The relationships of BMI, FMI, and FMP to lipid profiles For BMI categories, compared with non-overweight youth, youth with overweight (including obesity) had a higher TC, TG and LDL, lower HDL levels, and higher prevalence of each specific abnormal lipid $(P<0.01$, Supplementary Table S1 (online)). For FMI categories, significant differences of lipid profiles were also observed between youth with and without excess fat $(P<0.01$, Supplementary Table S2 (online)).

Correlations and linear regressions showed that BMI and FMI were positively associated with plasma TC, LDL-C, and TG, and inversely associated with $\mathrm{HDL}-\mathrm{C}$ in both genders (all $z$-scores, $P<$ 0.01 , Figs 1 and 2, Table 2). The standard multi-linear regression coefficients (beta) of BMI $z$-score and FMI z-score for each lipid profiles (except for HDL-C) were consistently lower in girls than in boys ( $P$ for interaction $<0.05$ ). Among boys, the adjusted standard regression coefficients of FMI $z$-score for TC, LDL-C, and TG were higher than that of BMI $z$-score $(P<0.01)$, but not for HDL-C $(P>$ 0.05 ). Differences between increments per 1 unit of FMI $z$-score and BMI $z$-score for TC, LDL-C, TG, and HDL-C (all $z$-scores) were $0.062,0.054,0.048$, and 0.01 , respectively. Whereas among girls, BMI $z$-score was substantially similar to FMI $z$-score on the association strength with lipid profiles (Table 2). After mutual adjustment, beta coefficients of BMI $z$-score with TC, LDL-C, and TG, FMI z-score with HDL were substantial reversed or attenuated in both genders (Supplementary Table S3 (online)). The 

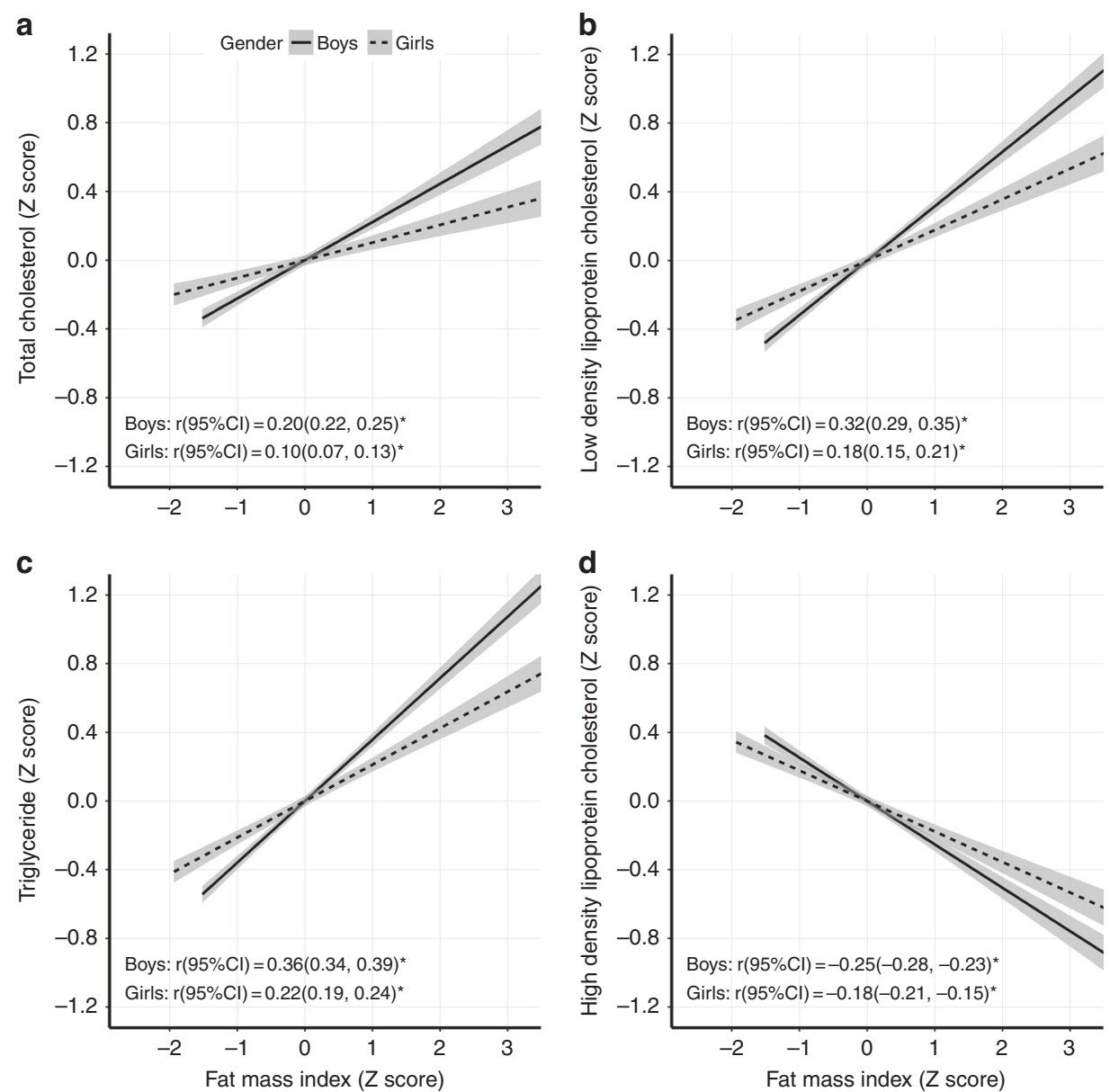

Fig. 2 Plot of relationships among fat mass index and lipid profiles by sex in Chinese children and adolescents aged 6-18 years. Fat mass index shown in relationship to total cholesterol (a), low-density lipoprotein cholesterol (b), triglyceride (c), and high-density lipoprotein cholesterol (d). Solid lines denote boys, dotted lines denote girls, shading indicate $95 \% \mathrm{Cl} . r(95 \% \mathrm{Cl})$, correlation coefficient and $95 \% \mathrm{Cl}$, ${ }^{*}$ all $P<0.05$. For triglyceride, $z$-score of log-transformed values

associations of BMl $z$-score, and FMI $z$-score with TC and LDL-C were stronger in the older (boys $\geq 11$ years, girls $\geq 10$ years) than in the younger age group (boys $<11$ years, girls $<10$ years) ( $P$ for interaction $<0.05)$. The differences between increments per 1 unit of FMI $z$-score and BMI $z$-score for lipid profiles (except for HDL-C) in older boys were greater than in younger boys (Supplementary Table S4 (online)).

Moreover, analyses of overweight and excess fat with specific abnormal lipid profiles using log-binomial regression models and ROCs revealed consistent results. The prevalence ratios (PRs) and area under curves (AUCs) of excess fat classified by FMI for specific abnormal lipid profiles (except for HDL-C) were higher than overweight classified by BMI in boys but not in girls (Tables 3 and 4). In boys, the AUCs for detecting children with abnormal TC, LDL-C, and TG of FMI-based excess fat were 3.9\%, 5.6\%, and $2.8 \%$ higher than of BMl-based overweight, respectively, all $P<0.01$.

All these results were almost identical when using FMP instead of FMI (Tables 2-4, Supplementary Figure S2 (online), Supplementary Tables S2-S4 (online)).

Associations between obesity types and specific abnormal lipid profiles

Among boys, compared those with non-overweight (classified by $\mathrm{BMI}$ ) and non-excess fat (classified by FMI), after adjusting for confounders, excess fat boys without overweight demonstrated significantly increased risks of having high TC, LDL, and TG (PRs: $3.07,4.72$, and 4.02 , all $P<0.05$ ), but almost no risk increased in overweight boys without excess fat (PRs: $0.65,0.85$, and 1.65, all $P>0.05)$. In contrast, those with overweight or excess fat showed similar prevalence rates among girls (Table 5). Again, analyses that used FMP instead of FMI to define excess fat gave similar but slightly weak results (Supplementary Table S5 (online)).

\section{DISCUSSION}

In this large nationwide cross-sectional study, we explored whether FMI and FMP were superior over BMI in identifying children with abnormal lipid profiles. Our findings indicated that FMI and FMP exhibited stronger associations to TC, LDL, and TG, suggesting body fat indicators were better than BMI in identifying abnormal lipid profiles in boys and were roughly equivalent to BMI in girls. In addition, the associations of BMI, FMI, and FMP with lipid profiles were more closely in boys than in girls.

Compelling epidemiologic studies ${ }^{6-9,16,27-29}$ show youths with overweight or excess fat are more likely to have abnormal lipid profiles, which can be partially explained by the increased burden of adiposity-related insulin resistance and leptin resistance. ${ }^{30,31}$ Unfortunately, previous studies mostly used weight status indicators $^{6-8,28,29}$ to assess adiposity and did not evaluate whether body fat indexes were better than weight status indicators to discriminate children with dyslipidemia. In 2015, a Brazilian study suggested BMI had little utility as screening tools for dyslipidemia in children. ${ }^{29}$ Recently, another cross-sectional study involving 840 children and adolescents (6-18 years) from Brazil reported that 
Table 2. Estimated increments in each lipid levels per 1 unit of BMI, FMl, and FMP (all z-scores) in boys and girls

\begin{tabular}{|c|c|c|c|c|}
\hline & \multicolumn{2}{|l|}{ Boys } & \multicolumn{2}{|l|}{ Girls } \\
\hline & $\beta$ & $P$-value & $\beta$ & $P$-value \\
\hline \multicolumn{5}{|l|}{ TC (z-score) } \\
\hline BMI (z-score) & 0.13 & $<0.001$ & 0.05 & 0.001 \\
\hline FMI (z-score) & $0.19^{*}$ & $<0.001$ & 0.07 & $<0.001$ \\
\hline FMP (z-score) & $0.21^{*}$ & $<0.001$ & $0.09^{*}$ & $<0.001$ \\
\hline \multicolumn{5}{|l|}{ LDL-C (z-score) } \\
\hline BMI (z-score) & 0.23 & $<0.001$ & 0.12 & $<0.001$ \\
\hline FMI (z-score) & $0.29^{*}$ & $<0.001$ & 0.14 & $<0.001$ \\
\hline FMP (z-score) & $0.29^{*}$ & $<0.001$ & 0.15 & $<0.001$ \\
\hline \multicolumn{5}{|l|}{ TG $(z \text {-score })^{\mathrm{a}}$} \\
\hline BMI (z-score) & 0.31 & $<0.001$ & 0.19 & $<0.001$ \\
\hline FMI (z-score) & $0.35^{*}$ & $<0.001$ & 0.21 & $<0.001$ \\
\hline FMP (z-score) & 0.33 & $<0.001$ & 0.18 & $<0.001$ \\
\hline \multicolumn{5}{|l|}{ HDL-C (z-score) } \\
\hline BMI (z-score) & -0.28 & $<0.001$ & -0.20 & $<0.001$ \\
\hline FMI (z-score) & -0.27 & $<0.001$ & -0.19 & $<0.001$ \\
\hline FMP (z-score) & $-0.23^{*}$ & $<0.001$ & $-0.13^{*}$ & $<0.001$ \\
\hline \multicolumn{5}{|c|}{$\begin{array}{l}\text { Models adjusted for age, study center, smoking, alcohol drinking, diet, and } \\
\text { physical activity } \\
B M I \text { body mass index, FMI fat mass index, FMP fat mass percentage, } H D L-C \\
\text { high-density lipoprotein cholesterol, } L D L-C \text { low-density lipoprotein choles- } \\
\text { terol, } T C \text { total cholesterol, } T G \text { triglycerides } \\
{ }^{a} z-s c o r e \text { of log-transformed values } \\
{ }^{*} P<0.05 \text {, indicating significantly different beta coefficients between FMI or } \\
F M P \text { and BMI }\end{array}$} \\
\hline
\end{tabular}

Table 3. Estimated PRs for specific abnormal lipid profiles of overweight or excess fat classified by BMI, FMl, and FMP in boys and girls

\begin{tabular}{|c|c|c|c|c|}
\hline & \multicolumn{2}{|l|}{ Boys } & \multicolumn{2}{|l|}{ Girls } \\
\hline & $\mathrm{PR}$ & $95 \% \mathrm{Cl}$ & $\mathrm{PR}$ & $95 \% \mathrm{Cl}$ \\
\hline \multicolumn{5}{|l|}{ High TC } \\
\hline Classified by BMI & 1.51 & $1.15-1.99$ & 1.27 & $0.95-1.69$ \\
\hline Classified by FMI & 2.20 & $1.67-2.90$ & 1.26 & $0.97-1.63$ \\
\hline Classified by FMP & 2.20 & $1.67-2.89$ & 1.31 & $1.00-1.70$ \\
\hline \multicolumn{5}{|l|}{ High $L D L-C$} \\
\hline Classified by BMI & 2.55 & $1.84-3.55$ & 1.94 & $1.44-2.60$ \\
\hline Classified by FMI & 3.98 & $2.83-5.59$ & 1.70 & $1.29-2.25$ \\
\hline Classified by FMP & 3.76 & $2.70-5.24$ & 1.55 & $1.17-2.06$ \\
\hline \multicolumn{5}{|l|}{ High TG } \\
\hline Classified by BMI & 4.07 & $3.30-5.03$ & 2.22 & $1.81-2.73$ \\
\hline Classified by FMI & 5.09 & $4.12-6.29$ & 2.24 & $1.83-2.74$ \\
\hline Classified by FMP & 4.50 & $3.68-5.50$ & 1.98 & $1.61-2.43$ \\
\hline \multicolumn{5}{|l|}{ Low HDL-C } \\
\hline Classified by BMI & 2.64 & $2.21-3.15$ & 2.37 & $1.89-2.97$ \\
\hline Classified by FMI & 2.42 & $2.03-2.88$ & 2.07 & $1.66-2.58$ \\
\hline Classified by FMP & 2.13 & $1.79-2.54$ & 1.94 & $1.55-2.43$ \\
\hline \multicolumn{5}{|c|}{$\begin{array}{l}B M I \text { body mass index, } F M I \text { fat mass index, } F M P \text { fat mass percentage, } H D L-C \\
\text { high-density lipoprotein cholesterol, } L D L-C \text { low-density lipoprotein choles- } \\
\text { terol, } T C \text { total cholesterol, } T G \text { triglycerides, } P R \text { prevalence ratio } \\
\text { Prevalence ratios were calculated by overweight or excess fat compared to } \\
\text { those who non-excess, classified by } B M I, F M I \text {, and FMP } \\
\text { Models adjusted for age, study center, smoking, alcohol drinking, diet, and } \\
\text { physical activity }\end{array}$} \\
\hline
\end{tabular}

Table 4. Performance of overweight or excess fat classified by BMI, FMI, and FMP in relation to specific abnormal lipid profiles based on ROC curve analysis

\begin{tabular}{llllll} 
Boys & & & & & \\
\hline SEN, & SPE, & PPV, & NPV, & AUC $(95 \%$ Cl), & $P$ value \\
$\%$ & $\%$ & $\%$ & $\%$ & $\%$ & $\begin{array}{l}\text { for AUC } \\
\text { difference }\end{array}$
\end{tabular}

\begin{tabular}{llllll} 
Girls & & & & & \\
\hline SEN, & SPE, & PPV, & NPV, & AUC $(95 \%$ Cl $)$, & $\begin{array}{l}P \text { value } \\
\text { for AUC } \\
\%\end{array}$ \\
$\%$ & $\%$ & $\%$ & $\%$ & difference
\end{tabular}

High TC

\begin{tabular}{lllllllllllll} 
Classified by BMI & 50.5 & 65.4 & 6.1 & 96.8 & $58.0(54.3-61.6)$ & Reference & 25.7 & 80.2 & 6.6 & 95.2 & $52.9(50.0-55.8)$ & Reference \\
Classified by FMI & 54.2 & 69.7 & 7.3 & 97.2 & $61.9(58.3-65.6)$ & 0.002 & 35.7 & 71.4 & 6.4 & 95.3 & $53.5(50.4-56.7)$ & 0.640 \\
Classified by FMP & 51.6 & 71.8 & 7.5 & 97.1 & $61.7(58.0-65.3)$ & 0.010 & 35.7 & 72.3 & 6.5 & 95.4 & $54.0(50.8-57.1)$ & 0.486 \\
High LDL-C & & & & & & & & & & & \\
Classified by BMI & 60.3 & 65.6 & 5.6 & 98.0 & $62.9(58.9-67.0)$ & Reference & 34.0 & 80.5 & 7.2 & 96.5 & $57.3(53.8-60.7)$ & Reference \\
Classified by FMI & 67.1 & 69.9 & 7.0 & 98.4 & $68.5(64.6-72.4)$ & $<0.001$ & 43.5 & 71.7 & 6.4 & 96.6 & $57.6(54.0-61.2)$ & 0.823 \\
Classified by FMP & 63.7 & 71.9 & 7.1 & 98.3 & $67.8(63.8-71.8)$ & 0.005 & 40.8 & 72.4 & 6.2 & 96.5 & $56.6(53.1-60.2)$ & 0.691 \\
High TG & & & & & & & & & & & \\
Classified by BMI & 69.5 & 68.0 & 16.9 & 96.0 & $68.7(66.3-71.2)$ & Reference & 38.9 & 81.4 & 14.6 & 94.2 & $60.1(57.5-62.8)$ & Reference \\
Classified by FMI & 70.6 & 72.3 & 19.3 & 96.3 & $71.5(69.1-73.8)$ & 0.002 & 48.1 & 72.6 & 12.5 & 94.5 & $60.3(57.6-63.1)$ & 0.852 \\
Classified by FMP & 65.4 & 74.2 & 19.1 & 95.8 & $69.8(67.3-72.2)$ & 0.341 & 42.7 & 73.0 & 11.5 & 94.0 & $57.9(55.2-60.6)$ & 0.048 \\
Low HDL-C & & & & & & & & & & & \\
Classified by BMI & 55.3 & 66.9 & 15.3 & 93.3 & $61.1(58.6-63.5)$ & Reference & 39.0 & 81.2 & 12.6 & 95.0 & $60.1(57.2-63.0)$ & Reference \\
Classified by FMI & 51.6 & 70.8 & 16.1 & 93.1 & $61.2(58.8-63.7)$ & 0.881 & 45.9 & 72.2 & 10.3 & 95.0 & $59.0(56.1-62.0)$ & 0.295 \\
Classified by FMP & 45.9 & 72.6 & 15.3 & 92.5 & $59.2(56.8-61.7)$ & 0.101 & 42.8 & 72.9 & 9.9 & 94.8 & $57.8(54.9-60.7)$ & 0.063 \\
\hline
\end{tabular}

$A U C$ area under curve, $B M I$ body mass index, FMI fat mass index, FMP fat mass percentage, $H D L-C$ high-density lipoprotein cholesterol, $L D L-C$ low-density lipoprotein cholesterol, NPV negative predictive value, PPV positive predictive value, $R O C$ receiver-operating characteristic curve, SEN sensitivity, SPE specificity, $T C$ total cholesterol, $T G$ triglycerides 
Table 5. Estimated prevalence, and PRs for specific abnormal lipid profiles according to different obesity types (combinations of BMI and FMI categories) in boys and girls

\begin{tabular}{|c|c|c|c|c|c|c|c|c|}
\hline & $\begin{array}{l}\text { BMI } \\
\text { Overweight }^{\mathrm{a}}\end{array}$ & $\begin{array}{l}\text { FMI } \\
\text { Excess fat }\end{array}$ & \multicolumn{3}{|l|}{ Boys } & \multicolumn{3}{|l|}{ Girls } \\
\hline \multicolumn{9}{|l|}{ High TC } \\
\hline & + & - & $8(2.3)$ & 0.62 & $0.30-1.27$ & $7(8.4)$ & 1.68 & $0.83-3.41$ \\
\hline & - & + & $15(8.8)$ & 2.99 & $1.78-5.03$ & $30(6.3)$ & 1.29 & $0.88-1.90$ \\
\hline & + & + & $88(7.1)$ & 1.98 & $1.48-2.65$ & $52(6.4)$ & 1.28 & $0.94-1.74$ \\
\hline & - & - & $43(1.6)$ & 1.00 & Reference & $103(3.3)$ & 1.00 & Reference \\
\hline & + & - & $5(1.4)$ & 0.85 & $0.34-2.13$ & $5(6.0)$ & 1.71 & $0.72-4.07$ \\
\hline & - & + & $15(8.8)$ & 4.77 & $2.70-8.42$ & $23(4.8)$ & 1.25 & $0.80-1.96$ \\
\hline & + & + & $83(6.7)$ & 3.78 & $2.63-5.44$ & $60(7.4)$ & 2.03 & $1.49-2.77$ \\
\hline \multicolumn{9}{|l|}{ High TG } \\
\hline & - & - & $94(3.4)$ & 1.00 & Reference & $166(5.4)$ & 1.00 & Reference \\
\hline & - & - & $174(6.4)$ & 1.00 & Reference & $151(4.9)$ & 1.00 & Reference \\
\hline & + & - & $38(11.0)$ & 2.15 & $1.55-2.98$ & $6(7.2)$ & 1.29 & $0.59-2.80$ \\
\hline & - & + & $22(12.9)$ & 1.63 & $1.08-2.47$ & $26(5.5)$ & 1.20 & $0.80-1.80$ \\
\hline & + & + & $204(16.5)$ & 2.90 & $2.40-3.50$ & 107 (13.1) & 2.54 & $2.01-3.22$ \\
\hline $\begin{array}{l}\text { BMI bod } \\
\text { total ch } \\
\text { Models } \\
\text { aOverwe } \\
\mathrm{b}_{n}(\%), \mathrm{r}\end{array}$ & $\begin{array}{l}\text { mass index, FM } \\
\text { esterol, TG trigl } \\
\text { ljusted for age, } \\
\text { ht including o } \\
\text { mber of specif }\end{array}$ & $\begin{array}{l}\text { is index, } F M \\
P R \text { prevalen } \\
\text { smoking, alc } \\
\text { nal lipid cas }\end{array}$ & $\begin{array}{l}\text { is percentag } \\
\text { nking, diet, } \\
\text { alence, \%) }\end{array}$ & cal ac & oprotein c & LD & op & erol, TC \\
\hline
\end{tabular}

FMP assessed by multi-frequency bioelectrical impedance analysis was superior to both BMI and waist-to-height ratio in identifying unfavorable lipid profiles among girls, ${ }^{27}$ but these indicators only classified obesity ( $\geq 95$ th) to evaluate non-HDL-C distribution. However, inconsistent results were observed in overweight or obese youths. ${ }^{32,33}$ In the present study, body fat was measured by DXA, which is one of the gold standards for body composition assessment and have a greater capacity to discriminate between fat and lean mass. ${ }^{34}$ Our results further indicated that DXAassessed FMI and FMP were more closely associated with TC, LDL$C$, and TG than BMI among boys.

The AAP and NHLBI guidelines ${ }^{10,11}$ recommend dietary or pharmacologic intervention for children based on TC and/or LDL-C thresholds, but rarely recommend any treatment for children with abnormal TG or HDL-C levels. Both Lee et al. ${ }^{17}$ and our study demonstrated that BMI was adequately capable of detecting abnormal TG and HDL-C in children, but not abnormal TC and LDL-C. Moreover, owing to the invasiveness and high cost of blood lipid examination, targeting the genuinely high-risk children for lipid screening is particularly critical. Conveniently, body fat measurements are widely used owing to the low cost and high expediency. ${ }^{35}$ Taken together, these findings suggest identifying children who need lipid examination and management based on body fat rather than weight status may be more precise and cost-effective.

We also observed that adiposity indexes were more strongly correlated with lipid profiles in boys than in girls, which were supported by previous studies. ${ }^{16,27}$ Girls versus boys were proven to have more subcutaneous fat and peripheral regional fat, ${ }^{14}$ which confer lower health risks than visceral fat and abdominal fat. ${ }^{36,37}$ In addition, girls also have a higher physiological level of estrogens that play important roles in preventing lipid disorder according to data in vitro and in vivo. ${ }^{38}$ Therefore, the differences in fat distribution and estrogen level between boys and girls may be responsible for gender differences in the association strength, and may also partly explain the better performance of FMI and FMP than BMI in identifying unfavorable lipid profile mainly in boys.

The relation between adiposity indicators and lipids also differed among age groups, as FMI and FMP versus BMI were more strongly associated with lipid profiles in older boys ( $\geq 11$ years, representing puberty and post-puberty). These findings are biologically plausible. First, lipid concentrations vary in the pubertal period. ${ }^{39}$ Second, previous and our studies show BMl is rising during the pubertal period in both genders, but FMI and FMP increase in girls and decrease in boys. ${ }^{14,15}$ These results suggest BMI is more inappropriate to evaluate adiposity and health risks in pubertal and postpubertal boys. The findings on sex and age differences suggest lipid screening in boys or older teens based on body fat status rather than BMI may be more useful.

However, both obesity and dyslipidemia are complex, multifactorial diseases with genetic, epigenetic and environmental components. ${ }^{40}$ Lipid concentrations in children and adolescents are not all related to obesity. ${ }^{18}$ It should also be noted HDL-C is usually inversely related to adiposity. However, why body fat did not perform better than BMI in associations with $\mathrm{HDL}-\mathrm{C}$ found in the present study may require further investigation.

Strengths of the current study include the large sample size, accurate measurement of body fat by DXA. In addition, the study included a nationwide sample of Chinese children with generalizability which had a wide range of BMI, FMI, FMP and lipid profiles. This study also has some potential limitations that deserve consideration. First, there is no clear definition of excess body fat in children. We defined high adiposity based on the CCACH 2013 to 2015 internal smoothed 75th percentile of FMI or FMP, which may be an inaccurate assumption. Second, data on pubertal stage was unavailable and cannot be accounted for, but 
we did use two age groups in an effort to reflect the pubertal period. Third, family history of hypercholesterolemia explains a large percentage of the variance in lipid concentrations, and the AAP and NHLBI screening guidelines ${ }^{10,11}$ for youths also mention the family history as a potential risk factor. Unfortunately, we did not obtain information on the family history, which may lead to overestimate the observed associations. Fourth, despite the large sample size and age span, we cannot examine the relationship between obesity indicators and lipid profiles in children aged 2-6 years.

In conclusion, DXA-assessed body fat is superior over BMI in identifying boys with abnormal lipid profiles. Further studies are warranted to prospectively evaluate the impact of lowering body fat on lipid disorders.

\section{CHINA CHILD AND ADOLESCENT CARDIOVASCULAR HEALTH (CCACH) COLLABORATION GROUP}

Hong Cheng ${ }^{3}$, Guimin Huang ${ }^{3}$, Dongqing $\mathrm{Hou}^{3}$, Fangfang $\mathrm{Chen}^{3}$, Wenpeng Wang ${ }^{3}$, Feng Xiong ${ }^{5}$, Jinghui Sun ${ }^{6}$, Wenqing Ding ${ }^{7}$, Weili Yan $^{8}$, Bo Xi ${ }^{9}$

${ }^{5}$ Department of Endocrinology, Children's Hospital of Chongqing Medical University, Chongqing, China; ${ }^{6}$ Department of Cardiovascular Medicine, The First Hospital of Jilin University, Changchun, Jilin, China; ${ }^{7}$ Department of Epidemiology, School of Public Health, Ningxia University, Ningxia, China; ${ }^{8}$ Department of Clinical Epidemiology, Children's Hospital of Fudan University, Shanghai, China and ${ }^{9}$ Department of Epidemiology, School of Public Health, Shandong University, Jinan, Shandong, China

\section{ACKNOWLEDGEMENTS}

We thank the other investigators, the staff, and the participants of the $\mathrm{CCACH}$ study for their valuable contributions. The study was supported by grants from the "Twelfth Five Year Plan" of the China National Science and Technology (2012BAI03B03), the National Key Research and Development Program of China (2016YFC1300101, 2016YFC0900602), and the Beijing Training Project for the Leading Talents in Science and Technology (2011 $\mathrm{L} 07)$

\section{AUTHOR CONTRIBUTIONS}

H.L., J.M., T.H., and Y.Y. conceptualized the study concept and design. J.M., J.L., Y.Y., and X.Z. conducted research; H.L., J.M., and P.X. analyzed the data; and H.L., J.M., T.H., and Y.Y. drafted the manuscript. All authors were involved in writing the paper and had final approval of the submitted and published versions.

\section{ADDITIONAL INFORMATION}

The online version of this article (https://doi.org/10.1038/s41390-019-0287-x) contains supplementary material, which is available to authorized users.

Competing interests: The authors declare no competing interests.

Publisher's note: Springer Nature remains neutral with regard to jurisdictional claims in published maps and institutional affiliations.

\section{REFERENCES}

1. Joshi, S. M. et al. Tracking of cardiovascular risk factors from childhood to young adulthood - the Pune Children's Study. Int. J. Cardiol. 175, 176-178 (2014).

2. Juhola, J. et al. Tracking of serum lipid levels, blood pressure, and body mass index from childhood to adulthood: the Cardiovascular Risk in Young Finns Study. J. Pediatr. 159, 584-590 (2011).

3. Haney, E. M. et al. Screening and treatment for lipid disorders in children and adolescents: systematic evidence review for the US Preventive Services Task Force. Pediatrics 120, e189-e214 (2007).

4. Gazzola, K., Reeskamp, L. \& van den Born, B. J. Ethnicity, lipids and cardiovascular disease. Curr. Opin. Lipidol. 28, 225-230 (2017).

5. Eslami, A. et al. Sex-specific incidence rates and risk factors of premature cardiovascular disease. A long term follow up of the Tehran Lipid and Glucose Study. Int. J. Cardiol. 227, 826-832 (2017).

6. Centers for Disease Control and Prevention (CDC). Prevalence of abnormal lipid levels among youths --- United States, 1999-2006. MMWR Morb. Mortal Wkly Rep. 59, 29-33 (2010).
7. Nogueira-de-Almeida, C. A. \& Mello, E. D. Correlation of body mass index Z-scores with glucose and lipid profiles among overweight and obese children and adolescents. J. Pediatr. 94, 308-312 (2017).

8. Boyd, G. S. et al. Effect of obesity and high blood pressure on plasma lipid levels in children and adolescents. Pediatrics 116, 442-446 (2005).

9. Lipsky, L. M. et al. Body mass index and adiposity indicators associated with cardiovascular biomarkers in youth with type 1 diabetes followed prospectively. Pediatr. Obes. 12, 468-476 (2017).

10. Daniels, S. R. \& Greer, F. R. Committee on N. Lipid screening and cardiovascular health in childhood. Pediatrics 122, 198-208 (2008).

11. Expert Panel on Integrated Guidelines for Cardiovascular Health and Risk Reduction in Children and Adolescents, National Heart, Lung, and Blood Institute. Expert panel on integrated guidelines for cardiovascular health and risk reduction in children and adolescents: summary report. Pediatrics 128(Suppl 5), S213-S256 (2011).

12. Mechanick, J. I., Hurley, D. L. \& Garvey, W. T. Adiposity-based chronic disease as a new diagnostic term: the American Association of Clinical Endocrinologists and American College of Endocrinology Position Statement. Endocr. Pract. 23, 372-378 (2017).

13. Romero-Corral, A. et al. Accuracy of body mass index in diagnosing obesity in the adult general population. Int J. Obes. 32, 959-966 (2008).

14. Wells, J. C. Sexual dimorphism of body composition. Best. Pract. Res. Clin. Endocrinol. Metab. 21, 415-430 (2007).

15. Borrud, L. G. et al. Body composition data for individuals 8 years of age and older: U.S. population, 1999-2004. Vital. Health Stat. 11, 1-87 (2010).

16. Lamb, M. M. et al. Association of body fat percentage with lipid concentrations in children and adolescents: United States, 1999-2004. Am. J. Clin. Nutr. 94, 877-883 (2011).

17. Lee, J. M. et al. Poor performance of body mass index as a marker for hypercholesterolemia in children and adolescents. Arch. Pediatr. Adolesc. Med. 163 716-723 (2009).

18. Daniels, S. R. Lipid concentrations in children and adolescents: it is not all about obesity. Am. J. Clin. Nutr. 94, 699-700 (2011).

19. Liu, J. et al. Bone mineral density reference standards for Chinese children aged 3-18: cross-sectional results of the 2013-2015 China Child and Adolescent Cardiovascular Health (CCACH) Study. BMJ Open 7, e014542 (2017).

20. Dong, $H_{\text {., }}$ et al. Alarming trends in ideal cardiovascular health among children and adolescents in Beijing, China, 2004 to 2014. Int. J. Cardiol. 231, 264-270 (2017).

21. de Onis, M. et al. Development of a WHO growth reference for school-aged children and adolescents. Bull. World Health Organ. 85, 660-667 (2007).

22. Baim, S. et al. Official positions of the International Society for Clinical Densitometry and executive summary of the 2007 ISCD Pediatric Position Development Conference. J. Clin. Densitom. 11, 6-21 (2008).

23. $\mathrm{Ma}, \mathrm{H}$. M. et al. Pubertal development timing in urban Chinese boys. Int. J. Androl. 34, e435-e445 (2011).

24. Sun, Y. et al. National estimates of the pubertal milestones among urban and rural Chinese girls. J. Adolesc. Health 51, 279-284 (2012).

25. Johnson, R. W. An introduction to the bootstrap. Teach. Stat. 23, 49-54 (2001).

26. Deddens, J. A. \& Petersen, M. R. Approaches for estimating prevalence ratios. Occup. Environ. Med. 65, 501-506 (2008).

27. Oliosa, P. R., et al. Body fat percentage is better than indicators of weight status to identify children and adolescents with unfavorable lipid profile. J. Pediatr. (RioJ). 95, 112-118 (2019). https://doi.org/10.1016/j.jped.2017.11.003.

28. Bijari, B. et al. The relationship between serum lipids and obesity among elementary school in Birjand: a case control study. J. Res Health Sci. 15, 83-87 (2015).

29. de Quadros, T. M. B. et al. Predictive capacity of anthropometric indicators for dyslipidemia screening in children and adolescents. J. Pediatr. 91, 455-463 (2015).

30. Pinhas-Hamiel, O. et al. Lipid and insulin levels in obese children: changes with age and puberty. Obesity 15, 2825-2831 (2007).

31. Jois, A. et al. Relationship of high leptin levels with an adverse lipid and insulin profile in 6-8 year-old children in Spain. Nutr. Metab. Cardiovasc. Dis. 25, 1111-1116 (2015).

32. Bohn, B. et al. BMI or BIA: is body mass index or body fat mass a better predictor of cardiovascular risk in overweight or obese children and adolescents? A German/Austrian/Swiss Multicenter APV analysis of 3,327 children and adolescents. Obes. Facts 8, 156-165 (2015).

33. Araujo, A. J., Santos, A. C. \& Prado, W. L. Body composition of obese adolescents: association between adiposity indicators and cardiometabolic risk factors. J. Hum. Nutr. Diet. 30, 193-202 (2017).

34. Bauer, J. et al. Dual-energy X-ray absorptiometry prediction of adipose tissue depots in children and adolescents. Pediatr. Res. 72, 420-425 (2012). 
Body fat indicators perform better than body mass index in identifying...

$\mathrm{H}$ Li et al.

624

35. Lemos, T. \& Gallagher, D. Current body composition measurement techniques. Curr. Opin. Endocrinol. Diabetes Obes. 24, 310-314 (2017).

36. Min, K. B. \& Min, J. Y. Android and gynoid fat percentages and serum lipid levels in United States adults. Clin. Endocrinol. 82, 377-387 (2015).

37. Elffers, T. W. et al. Body fat distribution, in particular visceral fat, is associated with cardiometabolic risk factors in obese women. PLoS ONE 12, e0185403 (2017)
38. Pellegrini, M. et al. Role of the sex hormone estrogen in the prevention of lipid disorder. Curr. Med. Chem. 21, 2734-2742 (2014).

39. Jolliffe, C. J. \& Janssen, I. Distribution of lipoproteins by age and gender in adolescents. Circulation 114, 1056-1062 (2006).

40. Klop, B., Elte, J. W. \& Cabezas, M. C. Dyslipidemia in obesity: mechanisms and potential targets. Nutrients 5, 1218-1240 (2013). 